\title{
Performance Enhancement of Mammogram Preprocessing for Breast Cancer Detection using Improved Successive Mean Quantization Technique
}

\author{
Pramod Patil ${ }^{1}$, Dr. S. Kotrappa ${ }^{2}$ \\ ${ }^{1}$ Research Scholar, Visveswaraya Technological University, Belagavi, Karnataka,India, \\ pramodkumar.patil@gmail.com \\ ${ }^{2}$ Professor, KLE's, Dr. M.S. Sheshgiri College of Engineering and Technology, VTU, Belagavi, Karnataka, India, \\ kotrappa06@gmail.com
}

\begin{abstract}
In the recent years the rise in cancer cases are at the peak and among different cancer sites the breast cancer is leading the race. It stood second most dangerous cancer among all cancer sites and it is a leading type of cancer among women's and also leading cause of cancer deaths among women's now a days. The thorough studies said that the survival rate of breast cancer is quite good but when it is detected early stage. In this paper we have highlighted the different obstacles that might leads the misprediction by the concern due to many types of artifacts and noise present in the mammogram and it is the main reason of misprediction and accuracy degradation. The paper presents the Improved Successive Mean Quantization Techniques (SMQT) with top hat transform, exponentiation and histogram enhancement to improve the quality of image with accurate region of interest and intensity image enhancement will play to vital role. Proposed model will give better accuracy when tested in MIAS and DDSM dataset it gives approximately 93 to 95 percent accuracy in all mammograms and average overlap ratio is grater that $85 \%$ in all tested mammograms.
\end{abstract}

Key words : Breast Cancer, Mammography, Successive Mean Quantization Technique, Image Binarization, top hat transform, Histogram enhancement

\section{INTRODUCTION}

Breast Cancer becomes second most deadly cancer all over the world, in some developed countries Breast Cancer will become a leading type of cancer in coming days. It is responsible for $23 \%$ of overall cancer cases and also it makes up $14 \%$ of all newly investigated cases and deaths. It becomes most common type of cancer in women's all over the world. According to World Health Organization (WHO) and International Agency of Research on Cancer (IARC) the estimated breast cancer cases in India in 2020 is near about 1.72 lakh new cancer cases are predicted and 92000 deaths are estimated due to breast cancer only. The same estimate likely to grow up to 2.2 lakh cases by 2030 [1] and the primary causes of the disease are still having a big question mark but the advancements in the modalities, Computer Aided Detection (CADe) and Computer Aided Diagnosis (CADx) are playing a vital role in the prognosis of breast cancer at early stage.

Research and Development in the fields of breast cancer research it is precisely found that the survival rate in the breast cancer category is quite good but when it is diagnosed at early stage only. For screening of breasts the different modalities available are Digital Mammography, Magnetic Resonance Imaging (MRI) or Digital Tomography type of screening is available now. The Digital Mammography is widely used and effective method for the early stage diagnosis of any kind of breast abnormalities present in the breast below 50 years of age. A systematic review came in 2015 and it is observed that the Digital Mammography reduces the mortality rate of breast cancer by around $20 \%$ in the women who are at the average risk of getting breast cancer development [2]. Digital Mammography is low radiation X-Ray of the breast which will produce image of the breast on a film [3].

Oncologist precisely suggest the mammography which is better than self-examination and accurate diagnosis and it also better to identity better before felt physically [4]. Mammography is aimed to detect the non-homogeneous type of cells and micro symptoms of breast cancer according to survey the early presence of microcalcification on digital mammogram is first sign of getting future development of breast cancer. Microcalcification is tiny calcium deposits that will appear on mammogram[5]. These signs are appears as visual threshold among the specific abnormality or between some background tissues and the micro signs are seems like microcalcification, masses, architectural distortion or asymmetry. In recent days CADe and CADx has been evolved with the many advanced mechanisms to detect any types of first sight signs or abnormalities from the digital scans.

The MLO view is very crucial for the processing but it also comes with the obstacles (Artifacts) which are likely to be not important for the processing mammogram these extra parts are labels, names, dates, names and pectoral muscles. These artifacts sometimes appear as bright as the tumor and it also appear with the same intensity like tumor has. 
So that sometimes it lowers the accuracy rate of detection and it also increase the processing time [6], [7]. Images are also prone to have different kind of noise or unwanted elements that may leads towards misconceptions in accuracy and increasing processing time. The noise present in the image which may be having similar features like tumor microcalcification so that enhancement of image is also important to get the clear vision of segments to process.

Figure 1. shows the sample mammogram in the both types of views the sample mammogram is for right breast and taken in CC and MLO view this both views are essential to analyze the complete anatomy of the breast. MLO view is more preferred over $\mathrm{CC}$ view as it covers the more visualization area for breast tissue which is present in upper quadrant.
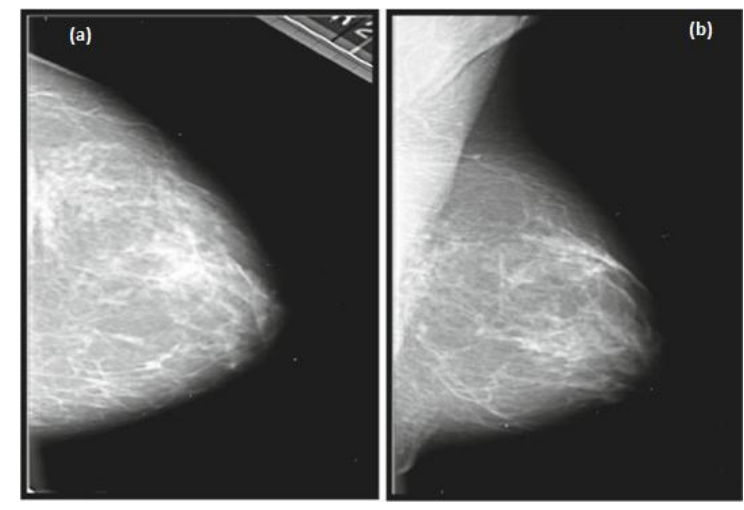

Figure 1: Mammogram of breast (a) Craniocaudal View (CC) (b) Mediolateral View (MLO)

In order to this preprocessing is a decisive phase for making mammogram easier for analysis of normal and any kind of abnormal conditions. Many times these types of medical scans may contain noise which will affect the accuracy. The image preprocessing must have first noise removal if any kind of noise is there and image enhancement for making mammogram vision lore likely to read accurately. In the mammogram some principal information may get blurred due to grey scale transmission the histogram transformation may transform image into uniform distribution. Firstly it performs the transformation of the original mammogram by applying separation of gray scale intervals into two parts and then reduce the intervals for enhancing the contrast level in the image[8]. This technique is mainly classify into following types,

1) Frequency domain.

2) Spatial domain.

3) Combining spatial and frequency domains.

These techniques are effectively implements based on their 4 types of features such as, region based, feature based, fuzzy based and conventional based. Pectoral muscle removal approaches are based on two methods Line detection and Intensity based. Intensity driven Approaches employ the intensity value of the Parenchyma in the breast is always smaller than in the pectoral muscle. These methods are based on pixel intensities [9-14] and image histograms [15-17]. Line detection approaches are based on the fact that the pectoral muscle is always present in the upper left / right corner of the mammography image and it has a triangular geometry.

In this paper novel approach for image preprocessing with pectoral noise removal from mammogram is presented and also method performs well in noise reduction, removal of background and artifacts done well.

Here in this study for our experimental work we have used publicly available most popular dataset Mammographic Image Analysis Society (MIAS)[18] and Digital Database for Screening Mammography dataset (DDSM) .

This paper is organised as, section 2 work done by different researchers has given, Section 3 presents the gaps analysis section 4 contains the proposed work the framework and proposed model will be discussed in details, Section 5 discuss on results and discussion with performance measure and Section 6 performance evaluation section 7 discusses conclusion of work section 8 depicts conflict of interest and section 9 has acknowledgement and section 10 has references used for this research.

\section{RELATED WORK}

S. Tripathy et al. [19] The study used histogram equalization and CLAHE for the effective analysis of clinical mammograms. The percentage of highly contrasted pixels was evaluated with the suggested system. The study used a novel pre-processing method based on statistical features for the detection of breast image artifacts and threshold methods for the detection of breast mass border. This result could be effectively used for the advance analysis of the breast tumor as shown in the performance analysis of the state-of-the-art pre-processing techniques. Method obtained a contrast measurement of 0.021 and $\mathrm{CII}$ a contrast improvement index of 0.044 .

Ibrahim et al. [20] Presented an algorithm for the Pre-processing mammograms. It's using a two-dimensional Median filter for noise removal. Removal of artifacts and Background separation, thresholding is used. For the Contrast Improved Mammography Images, Band Limited equalization of histogram (BLHE) is used. Even though They pre-processed the mammography images, they skipped. Removal of the breast muscle.

Angayarkanni and Kumar [21] [22] Pre-processed breast images using Euclidean Distance the Transform (EDT) system.

Ramani et al. [23] discussed the different issues. Filters used to remove noise from the preprocessing step Mammograms.

Neeraj Shrivastava and Jyoti Bharti [24] The technique proposed in this paper has several stages of pre-processing Mammograms, this article focus on artifacts and background are removal by changing the original image to binary image using Otsu 's threshold. Medio-lateral view of the mammography images Two directions: left or right. Orientation detection is important for the pectoral muscle in the next step. Removal, which is done using the method of the line segment. Contrast is enhanced by limited contrast Adaptive histogram equalization, and noise is reduced by means of a median filter. 
Pramod Patil et al., International Journal of Emerging Trends in Engineering Research, 8(9), September 2020, 5149 - 5157

Ramani et al. [25] In this article authors discussed about different types of filters used in the noise removal process. Omer and Elfadil [26] In this article authors depicts preprocessing of mammographic images they applied auto cropping and label removal, for removal of impulse noise a median filter was applied. Contrast enhancement they have applied CLAHE and for pectoral muscle removal connected component labelling is used.

J Babu et al. [27] The SMQT extracted the information structure in an efficient manner. In this study, the capability of embedding was detected by non-zero DCT coefficients. In BSS, the cover image has been pre-processed in two steps, the embedding stage and the pre-processing stage. The study used the pre-processing stage to enhance contrast and other such manipulations on the corresponding cover images.

J Ma et al. [28] the study showed that the enhanced SMQT algorithm effectively reduced image noise and produced more detailed images. Apart from that, the contrasts and brightness are consistent in the visual effect to a high degree. Thus, the enhanced algorithm produces better results.

\section{GAP ANALYSIS}

The state of the art survey comes to the conclusion about the preprocessing of the input image is having equal importance as if it remains some misconceptions it will leads degrade in accuracy of detection of affected regions.

The preprocessed image must be,

1) It must be free from noise.

2) It must be having clear vision for bright regions and dark regions will be removed smoothly.

3) The brighter regions may has some kind of tissues which will develop a cancer so bright regions separate out who has any disorder.

4) Still preprocessing is having a scope to work for accurate region of interest selection so as to improve accuracy.

\section{PROPOSED WORK}

Pectoral muscle is an extra part of mammography Images that have a triangular shape and are always present either in the upper corner of the left or right corner. It's got a high intensity value compared to the parenchyma of the breast and almost the same intensity value as

a tumor in the abrasion image, which may result in incorrect mammography segmentation Pictures. Another issue with pre-processing mammograms is removing the background and the artifacts. The artifacts nothing but information on the image, like the date taken, views, etc. Generally, a mammogram is affected by noise. Therefore, noise reduction is also a challenge in pre-processing. In this paper, the different stages for pre-processing are presented.

The proposed flow of the work is depicted in Figure 2. The system will take input as a mammogram image generally here we have used images from the MIAS dataset then artifacts and background is to be removed as it affects the accuracy and also misleads the processing. The artifacts such as identification marks, opaque sign that affects the quality of the image. The artifacts removal and background clearance is very much essential in the medical imaging so that the system will get completely important information of mammogram only.

The image enhancement will improve the visualization of important information on mammogram this paper presents the improved Successive Mean Quantization Techniques [29][30]. The structural information on is broken down and these properties will be used in the local areas of the image to extract unwanted features. The local segments are presented in multiple ways,

In the proposed work, the image enhancement combines top hat transform and exponentiation operation. Then the histogram enhancement was used to enhance the process of microcalcification detection. The proposed hybrid method improved the accuracy of microcalcification detection compared to other existing methods.

The proposed method is designed by keeping image enhancement issues in mind [31] some common image enhancement challenges are,

1) Complex structure of breast anatomy and tissue density variations may occur. Dark small-scale lesions that could be fatal to the investigation.

2) Misidentification of microcalcification due to its small size, topology and which are initial predictor.

3) Malignancy signs such as masses, architectural distortion and asymmetry in breasts.

4) Low contrast regions where any of the malignancies are developed is hard to trace without enhancement and clear vision.

The proposed hybrid method is depicted in the Figure 2. The hybrid approach has compared with the existing one and results are compared the details are available in results and discussion section.

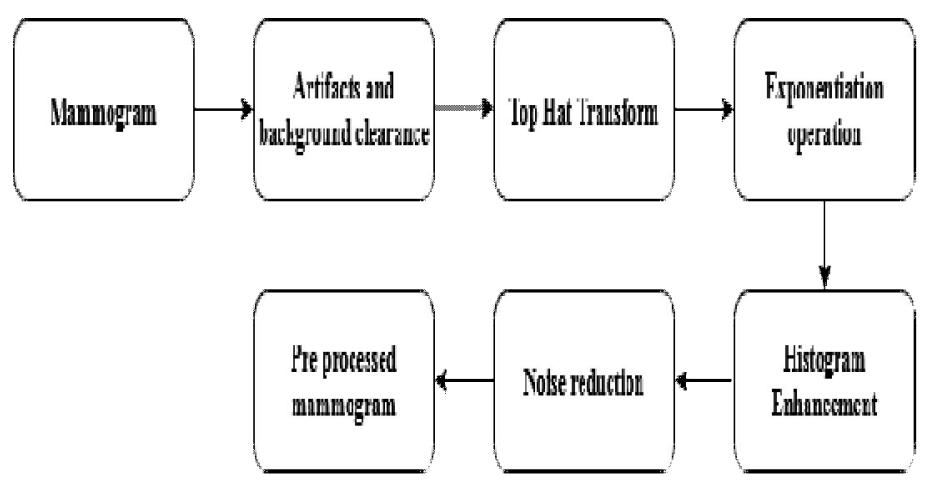

Figure 2: Flow of proposed improved SMQT model 


\subsection{Top Hat Transform}

Top hat transform is used to improve the contrast between images using morphological methods. Top hat transform is measured by subtracting the image from its original image [32].

In the Figure 3 it is depicted that the cross section of top hat transform and its transformation with the mathematical morphological parameters considered for the transform.

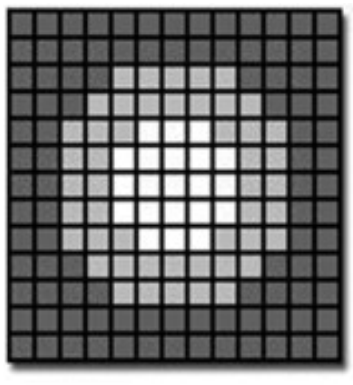

a

Figure 3: Top Hat Transformation

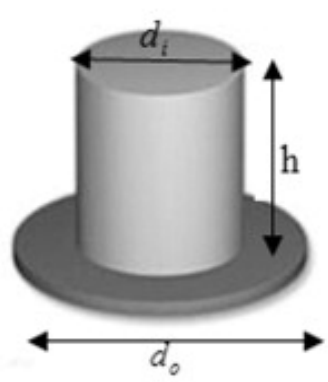

b
Consider $\mathrm{M}$ is the structuring elements and $\mathrm{N}$ as the original image that is represented by cartesian coordinates. The gray scale image dilation defines the maximum values added to the $\mathrm{M}$ image and it is presented as $\mathrm{M} \oplus \mathrm{N}$ so that the image boundaries will get clear visible. Similarly the grey scale erosion defines the maximum values subtracted from the current values of $\mathrm{M}$ structuring elements and the resultant is noted as $\mathrm{M} \ominus \mathrm{N}$. In the top hot transformation mainly this opening and closing two operations are performed.

The grey scale opening operation elucidates the erosion and the dilation operation having the same elements as $\mathrm{N}$.

It is represented as,

$$
\begin{aligned}
(\mathrm{M} \circ N)= & (\mathrm{M} \ominus \mathrm{N}) \oplus \mathrm{N} \quad--(4.1) \\
& (\mathrm{M} \cdot N)=(\mathrm{M} \oplus \mathrm{N}) \ominus \mathrm{N} \quad---(4.2)
\end{aligned}
$$

Mainly the opening operation considers the bright regions of the image and the closing focus on dark regions and when opening executes on the image and leaves the image background then the top hat transform removes the image background. Here equation 4.1 represents the opening operation and 4.2 represent the closing.

The two main types of top hat transform plays a crucial role such as, white top hat transform and black top hat transform. White top hat defines the fundamental difference between its opening operation by some structuring element and black top hat represent image and its difference between closing by some structuring element.

$$
\begin{aligned}
& \mathrm{h} 1=\mathrm{M}-(\mathrm{M} \circ N) \\
& \mathrm{h} 2=\mathrm{M}-(\mathrm{M} \cdot N)
\end{aligned}
$$

Here $\mathrm{h} 1$ represents white top hat transform and $\mathrm{h} 2$ represents black top hat and finally the contrast enhancement of the input image $\mathrm{M}$ is estimated by adding bright regions and removing dark regions form image and it is evaluated by,

$$
\mathrm{C}=\mathrm{M}+\mathrm{h} 1-\mathrm{h} 2
$$

Here $\mathrm{C}$ presents the contrast enhanced image and equation 4.5 represents the evaluation for contrast enhancement.

\subsection{Exponentiation operation}

After the morphological operation on input image the exponentiation is performed on the image it enhances the mammogram image. The $4^{\text {th }}$ order exponentiation is applied on the contrast enhance image.

$$
\mathrm{X}=C^{4}
$$

We obtain the $\mathrm{X}$ image which is exponent image with order 4 and then the proposed improved SMQT model applied and it uses the set theory operation to evaluate the mammogram image.

Let " $y$ " be a data coordinates and $\mathrm{S}(\mathrm{y})$ be a set of $|\mathrm{S}(\mathrm{y})|=\mathrm{S}$. The data coordinates value will represent as $\mathrm{V}(\mathrm{y})$. The data coordinates contain an arbitrary form that is $\mathrm{S}(\mathrm{y})$ be a vector. The proposed SMQT contains only one input parameter (S(y) $\rightarrow$ input set). From the input transform, the output set is defined as $\mathrm{T}(\mathrm{y})$ that had the same input form and the same size as the matrix.

The transformation is evaluated as,

$$
\operatorname{SMQT}_{L}: \mathrm{S}(\mathrm{y}) \rightarrow \mathrm{T}(\mathrm{y})
$$

The $S M Q T_{L}$ function is evaluated by using binary tree(BT). In BT the nodes are defined as Mean Quantization Units (MQU) and the MQU is evaluated by using three steps such as,

1) Mean calculation

2) Quantization

3) Split of the input image.

The evaluation takes place based on this three steps and BT concept.

\subsubsection{Mean calculation}

In Mean calculation, the threshold which is mean of the binary representation of the image is placed at the root node where the image binarization which is the robust method for the selection of the threshold of the image is used.

The probability of class occurrence is calculated for two pixel classes such as " $C_{1}$ " and " $C_{2}$ ".

$$
\begin{aligned}
& \omega_{1}=S_{r}\left(C_{1}\right)=\sum_{n=1}^{l} S_{n} \\
& \omega_{2}=S_{r}\left(C_{2}\right)=\sum_{i=l+1}^{M} S_{n}=1-\omega_{1}--(4.9)
\end{aligned}
$$

It calculates the average grey and class variance values, and then it determine the best threshold value with grayscale from 1 to $P$. The mean of pixels can be calculated by, 


$$
\bar{G}(n)=\frac{1}{|E|} \sum_{n \epsilon E} G(n)
$$

\subsubsection{Quantization}

In quantization process the image values in large extent is quantized using mean $\mathrm{x} \in \mathrm{E}$ where the comparison function is used as,

$$
\delta(G(n), \bar{G}(n))=\left\{\begin{array}{lr}
1, & G(x)>G(n) \\
0, & \text { else }
\end{array}\right.
$$

Let $\otimes$ denotes concatenation,

$$
Q(n)=\underset{x \in E}{\otimes} \delta(G(x), \bar{G}(n))
$$

It would be considered as the main output of MQU and quantized pixel sets.

\subsubsection{Split of the input image}

Thereafter quantization process, the derived input set is divided into two half's such as,

$$
\begin{aligned}
E_{0}(n) & =\{G(n) \leq \bar{G}(n), \forall n \in E\}--(4.13) \\
E_{1}(n) & =\{G(n)>\bar{G}(n), \forall n \in E\}-- \text { (4.14) }
\end{aligned}
$$

Here in binary representation it is iterative process for all layers but for one MQU the $E_{0}(n)$ is placed as left branch and $E_{1}(n)$ is placed as a right branch which is dependent on the structuring element value is less than or greater that root threshold placed at the root node. Figure 4. represents the single MQU unit.

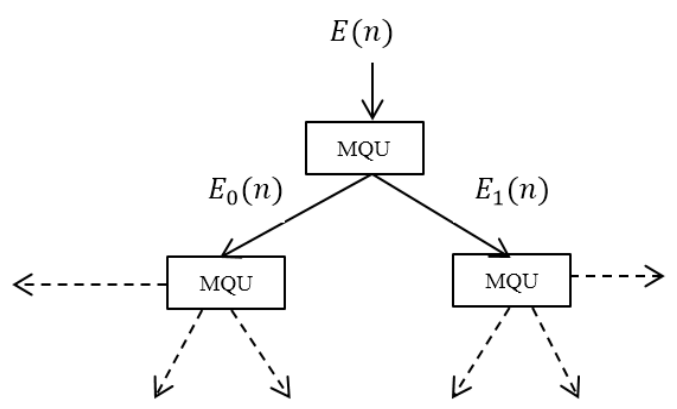

Figure 4. Single MQU unit's representation

Consider the $\mathrm{H}(l, \mathrm{n})$ output from the MQU operation in the binary tree, where $l=1,2, \ldots \ldots . \mathrm{M}$ is the current $\mathrm{n}=1,2, \ldots \ldots$. $2^{\wedge}(\mathrm{k}-1)$ at the level. In the proposed SMQT, Figure 5 defines the Binary MQU Tree.

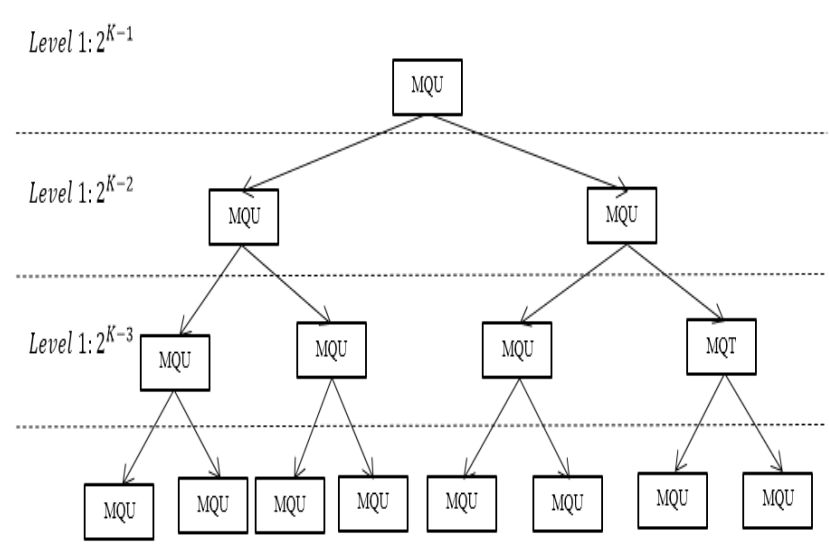

Figure 5. SMQT model binary tree representation of image.

Then the Output of SMQT is defined as

$S(n)=$
$\left\{n \mid G(n)=\sum_{i=1}^{K} \sum_{j=1}^{2^{i-1}} G\left(b_{i, j}\right) * 2^{K-1}, \forall n \in S, \forall b_{j, i} \in B_{i, j}\right\}$

\subsection{Histogram enhancement in proposed SQMT model.}

The histogram enhancement technique adjusts the image strength to an enhanced contrast level. The lower, focused, narrow image towards the grayscale increases the image quality with the histogram. The probability function is used to achieve the same probability as a normal histogram. The pixel intensity value input image contains the same value as the image output values to achieve the same pixel distribution. The distribution function increases the contrast between the image and the histogram. Equalization of histograms enhances multiple contrast images defined by close contrast values. This approach is used to analyse the image background and foreground whether it is dark or bright. The histogram is used on the basis of missing values of intensity and entropy for the dynamic range allocation. The new dynamic intensity range was evaluated in the input image from the original dynamic range.

The original dynamic range is represented as the " $\mathrm{S}$ " span and the new dynamic range is represented as the "range."

Let $l_{\mathrm{i}}$ and $\mathrm{u}_{\mathrm{i}}$ define the minimum and maximum intensity values of histogram.

$$
f_{i}(S(n))=S\left(l_{i}\right)+\left(S\left(u_{i}\right)-S\left(l_{i}\right)\right)--
$$

Histograms are generated on the basis of entropy. So, the original dynamic range of "S" depends on the entropy. When the sub-histogram is equalized to a small "S" value, the result will gain a poor contrast image. Let $\mathrm{X}$ defines a contrast enhanced image with different levels of discrete dynamic range. In addition, the proposed method uses entropy information for both the dynamic range sub histogram estimates and the division of histograms [33]. 
Pramod Patil et al., International Journal of Emerging Trends in Engineering Research, 8(9), September 2020, 5149 - 5157

$$
\begin{aligned}
& X\left(l_{i}\right)=\sum_{t=1}^{i-1} \text { range }_{t}+1 \\
& X\left(u_{i}\right)=\sum_{t=1}^{i} \text { range }_{t}
\end{aligned}
$$

In an enhanced image, the minimum $X\left(l_{i}\right)$ and maximum $X\left(u_{i}\right)$ intensities of sub-histogram are calculated.

Final contrast enhanced image represents as,

$$
C_{E}=f_{i}(S(n)) \text {---- (4.19) }
$$

$C_{E}$ will gives the final contrast enhancement image.

\section{RESULT AND DISCUSSION}

In the Results section, we described the pre-processing results of the proposed SMQT technique with histogram analysis. Five types of image abnormalities are used as input images to analyze the results and to obtain the enhanced image using the proposed method. The effectiveness of the proposed method combines morphological improvement and improved SMQT algorithm.

Table 1: Comparison of input image vs enhanced image with proposed SMQT model.
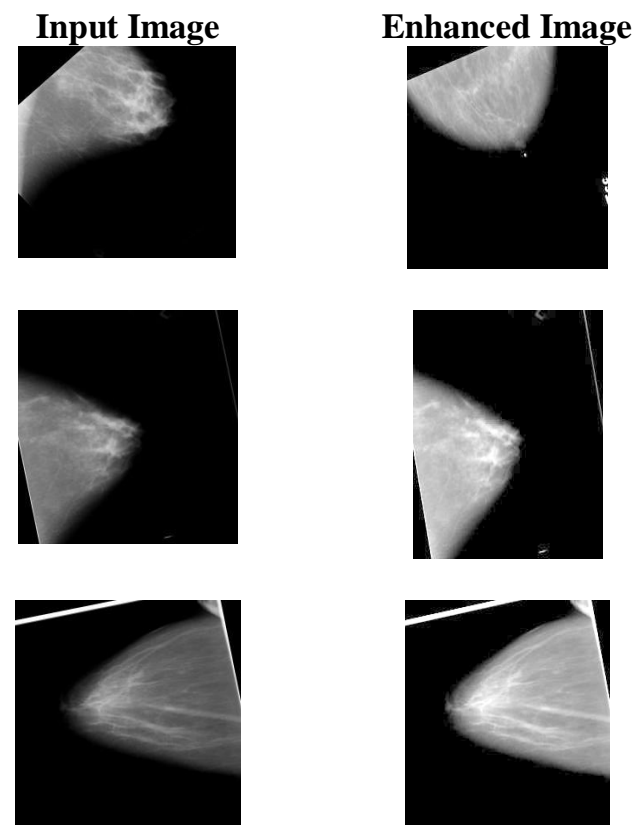

Table 1 shows the comparisons of the proposed improvement results for different types of image abnormalities. Enhanced image defines the image input after contrast enhancement by applying the proposed method. This image shows a better visibility of the proposed method compared to the poor effect of the input image on the detecting microcalcification. By applying the SMQT technique, it improves the readability of the breast region to identify abnormalities.

\subsection{Histogram Processing}

Histogram image defines the frequency of relevance of the existence of different gray levels in the original image. The histogram equalization compresses the image as pixel values that often occur in the image.

It is the most non-linear point and modifies the intensity of the image to improve the image contrast. The main objective of histogram equalization is to achieve a uniform distribution of the gray values on the input image [34].

Table 2: Comparison of input image vs enhanced image with proposed SMQT model.

\section{Input Histogram}
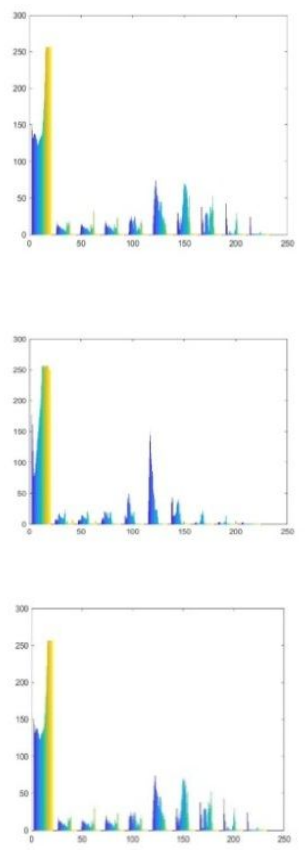

\section{Enhanced Histogram}
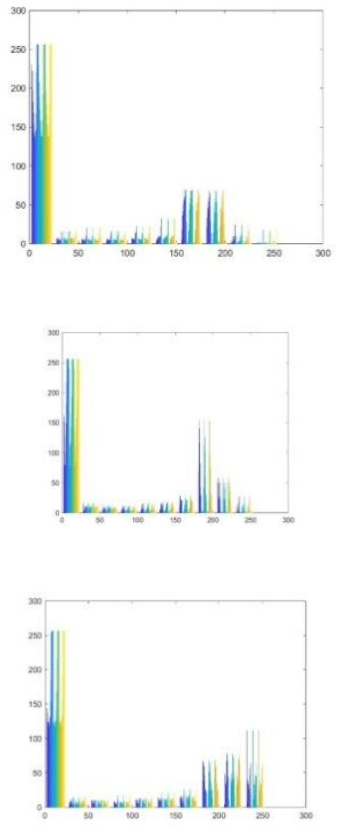

Table 2 represents the before histogram and after histogram equalization. The pixel values of the image appear infrequently and become less visible in the original image without a histogram. In the original image, the different parts of the image have a different gray intensity that will not be visible to the eyes.The increasing number of histograms improves the contrast of the image and can be visible to normal eyes that detect the full scale of the gray intensity [35][36].

\section{PERFORMANCE ANALYSIS}

For analyzing performance of the proposed model we have considered parameters as, overlapping ratio, accuracy, specificity, sensitivity, recall, precision, F score etc.

\subsection{0verlapping ratio}

From the MIAS dataset 25 microcalcification images and 55 images without microcalcification is selected randomly. The mammogram images applied with the proposed model and analyzed the performance. In the proposed method, the 
Pramod Patil et al., International Journal of Emerging Trends in Engineering Research, 8(9), September 2020, 5149 - 5157

bounding circle is drawn to detect the microcalcification region.

Table 3: Overlapping ratio comparison

\begin{tabular}{|l|c|c|}
\hline Image & Overlap ratio & Condition(>2/3) \\
\hline mdb009 & 93.80 & $\mathrm{Y}$ \\
\hline mdb035 & 98 & $\mathrm{Y}$ \\
\hline mdb043 & 100 & $\mathrm{Y}$ \\
\hline mdb057 & 89 & $\mathrm{Y}$ \\
\hline Mdb107 & 100 & $\mathrm{Y}$ \\
\hline Mdb137 & 96 & $\mathrm{Y}$ \\
\hline Mdb145 & 92 & $\mathrm{Y}$ \\
\hline Mdb163 & 86 & $\mathrm{Y}$ \\
\hline
\end{tabular}

Table 3 depicts the result of some images tested for overlapping ratio with proposed method and the result we can analyse all images are giving overlapping ratio greater than or equal to $85 \%$.

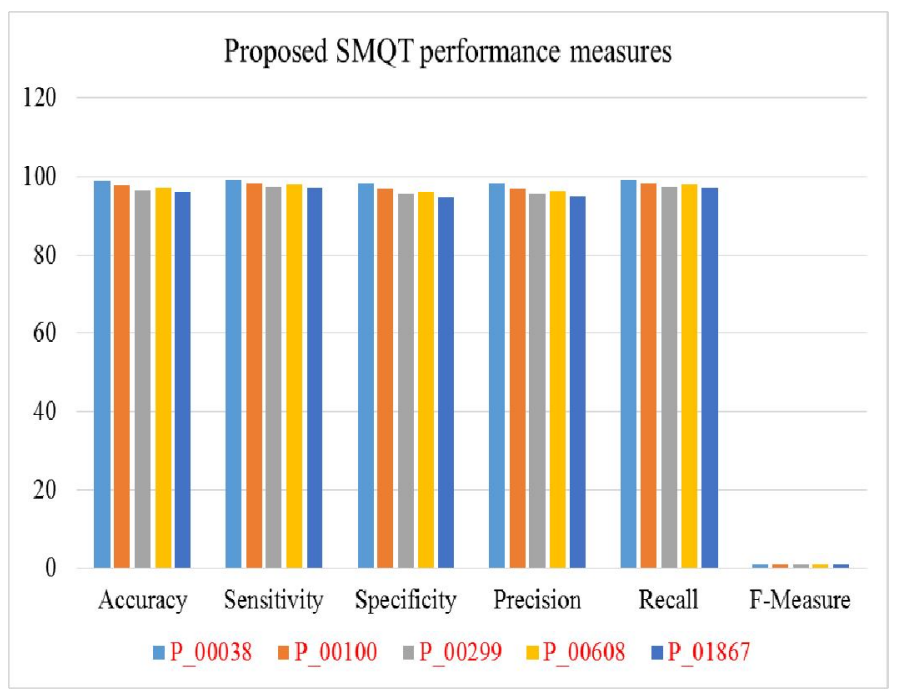

Figure 6: Performance measure of proposed SMQT model.

Figure 6. shows that the various performance measure calculated for this some images from DDSM dataset was considered. The results obtained with proposed model are quite better.

\subsection{Peak Signal-to-Noise Ratio (PSNR)}

The PSNR evaluates the peak among the two images in decibels. As the PSNR ratio augments, the image pixels will be de-speckling withappropriate improvisation with the deployment of filters. The PSNR can be calculated by,

$$
P S N R=\log _{10}\left(\frac{255}{M S E}\right)^{2}---(6.1)
$$

Figure 7 shows the Peak signal to noise ratio basically it is related with the quality of the image after compression and decompression and the results obtained are better in proposed SMQT model. Image mdb145 is having PSNR value 43.28. It builds the confidence in image quality.



Figure 7: Performance measure for PSNR

\subsection{Mean Square Error( MSE)}

MSE is basically related with the image quality such as it shows pixel variation in the image. MSE evaluation done by,

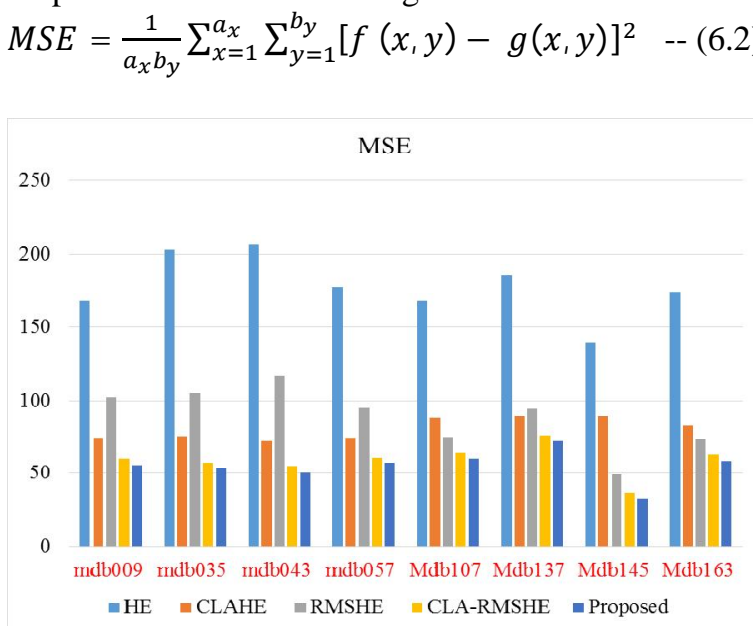

Figure 8: MSE evaluation

Figure 8. shows the comparison of MSE measurements with other existing methods such as HE, CLAHE, RMSHE, and CLA-RMSHE methods. This analysis proved that the proposed method had better MSE values for microcalcification detection in the database

\section{CONCLUSION}

This work is basically focuses on mammogram preprocessing for further analysis. The results are evaluated on MIAS and DDSM dataset. The mammogram is read in grayscale for processing and noise removal with pepper and salt noise removal. Then the artifacts and background is removed using the improved SMQT technique. SMQT is applied with top hat transform, exponentiation which will be 
Pramod Patil et al., International Journal of Emerging Trends in Engineering Research, 8(9), September 2020, 5149 - 5157

used to get the clear and enlarged view of mammogram then for more accuracy we have applied histogram enhancement to get the clear view of small disorders which will lead our work to improve accuracy over existing. The processed images are having average overlap ration greater than $85 \%$. The specificity and accuracy will be reached to $93 \%$ and $94 \%$ respectively but the performance enhancement is measured by using Peak signal to noise ration which shows image quality after preprocessing and mean square error ratio shows that the variation in the mammogram images.

However we are sure the proposed improved SMQT with histogram enhancement will improve the image preprocessing and it results into accurate prediction of early presence of breast cancer.

\section{ACKNOWLEDGEMENT}

At the outset, I would like to express deep gratitude to the both data set providers MIAS and DDSM for making mammographic data set available freely for research purpose. I would like to express my deep regards to my research supervisor Dr. S. Kotrappa sir for continuous motivation and timely guidelines during research journey.

\section{REFERENCES}

[1] "Cancer tomorrow." http://gco.iarc.fr/tomorrow/home (accessed Jul. 09, 2020).

[2] Y.-C. Tina Shih, W. Dong, Y. Xu, and Y. Shen, "Assessing the Cost-Effectiveness of Updated Breast Cancer Screening Guidelines for Average-Risk Women," Value in Health, vol. 22, no. 2, pp. 185-193, Feb.2019, doi: 10.1016/j.jval.2018.07.880.

[3] C. Printz, "ACS updates breast cancer screening guidelines," Cancer, vol. 122, no. 5, pp. 663-663, 2016, doi: 10.1002/cncr.29911.

[4] "InTech-digital_mammography.pdf." Accessed: Jul. 16, 2020. [Online]. Available: https://cdn.intechopen.com/pdfs/32008/InTe ch-Digital_mammography.pdf.

[5] Z. Chen, H. Strange, A. Oliver, E. R. E. Denton, C. Boggis, and R. Zwiggelaar, "Topological Modeling and Classification of Mammographic Microcalcification Clusters," IEEE Transactions on Biomedical Engineering, vol. 62, no. 4, pp. 1203-1214, Apr. 2015, doi: 10.1109/TBME.2014.2385102.

[6] D. N. Ponraj, P. Poongodi, and J. Manoharan, "A Survey on the Preprocessing Techniques of Mammogram for the Detection of Breast Cancer," undefined, 2011. /paper/A-Survey-on-the-Preprocessing-Tech niques-of-for-the-Ponraj-Poongodi/496b19d 56f0d3cbc5085f2b682dba151bc3daa29.

[7] A. C. C. -, A. B. -, A. M. -, and M. A. -, "An
Automatic-Pre-processing Method For Mammographic Images," JDCTA, vol. 4, no. 3, pp. 190-201, Jun. 2010, doi: 10.4156/jdcta.vol4.issue3.19.

[8] A. R. Mrunalini and J. Premaladha, "Performance Analysis of Image Enhancement Techniques for Mammogram Images," in Proceedings of the International Conference on ISMAC in Computational Vision and Bio-Engineering 2018 (ISMAC-CVB), Cham, 2019, pp. 1711-1723, doi: 10.1007/978-3-030-00665-5_158.

[9] Saltanat M, Hossain MA, Alam MS, "An efficient pixel value based mapping scheme to delineate pectoral muscle from mammograms", International Conference on Bio-Inspired Computing: Theories and Applications; 23-26 September 2010, Changsha China pp. 1510-1517.

[10] Roshan DY, Harada K., "A connected component labeling algorithm for grayscale images and application of the Algorithm on mammograms", Annual ACM Symposium on Applied Computing, Seoul Republic of Korea; 11-15 March 2007 pp. 146-152.

[11] Nagi J Kareem SA, Nagi F, Ahmed SK, "Automated breast profile segmentation for ROI detection using digital mammograms", Conference on Biomedical Engineering \& Sciences Kuala Lumper Malaysia, New York, Dec 2010.

[12] Liu L, Wang J., He K., "Breast density classification using histogram moments of multiple resolution histograms", International Conference on Biomedical Engineering and Informatics; 16-18 October 2010 Yantai China NewYork pp 146-149.

[13] Liu CC, Tsai CY, Liu J, Yu SS, “A pectoral muscle segmentation algorithm for digital mammograms using Otsu Thresholding and multiple regression analysis", Comput Math Appl 2012; 64: pp 1100- 1107

[14] Kurt B, Nabiyev VV, Turhan K, "A novel automatic suspicious mass regions identification using Havrda \& Charvat entropy and Otsu's N thresholding", Compu Meth Prog Bio 2014; 114: 349-360.

[15] David R, Arnau O, Joan M, Marta P, Joan E, "Breast Segmentation with pectoral muscle suppression on digital mammograms", Lecture Notes Computer Science 2005; 153-158.

[16] Thangavel K, Karanan M., "Computer aided diagnosis in digital mammograms: Detection of micro calcifications by meta heuristic algorithms", International Journal Artificial Intelligent machine Learning 2005; pp 29-40.

[17] Subashini T S, Ramalingam V, Palanivel S, "Pectoral muscle removal and detection of 
Pramod Patil et al., International Journal of Emerging Trends in Engineering Research, 8(9), September 2020, 5149 - 5157

masses in digital mammogram using CCL", International

Journal of Comput Appl 2010, pp 29-40.

[18] Suckling, J., Parker, J., Dance, D., Astley, S., Hutt, I., Boggis, C., \& Ricketts, I. (1810). Mammographic image analysis society (MIAS) database v1. 21 [dataset](2015).

[19] S. Tripathy and T. Swarnkar, "Unified preprocessing and enhancement technique for mammogram images," Procedia Computer Science, vol. 167, pp. 285-292, 2020

[20] Naglaa S. Ali Ibrahim, Naglaa F. Soliman, Mahmoud Abdallah, Fathi E. Abd El-Samie, "An Algorithm for Pre-processing and Segmentation of Mammogram Images", International Conference on Computer Engineering and Systems (ICCES) Cairo, Egypt, 20-21 Dec 2016 IEEE pp. 187-190.

[21] N. Angayarkanni and D. Kumar, "Euclidean Distance Transform (EDT) Algorithm Applied to Binary Image for Finding Breast Cancer", "2nd International Conference on Innovations in Information, Embedded and Communication System (ICIIECS) 2015 IEEE

[22] N. Angayarkanni and D. Kumar, "Euclidean Distance Transform (EDT) Algorithm Applied to Binary Image for Finding Breast Cancer", Biomedical \& Pharmacology Journal, Vol. 8(1), 407-411 (2015)

[23] R. Ramani, N. Suthanthira Vanitha, S. Valarmathy,"The Pre-Processing Techniques for Breast Cancer Detection in mammography Images", International Journal of Image, Graphics and Signal Processing, 2013 pp 47-54.

[24] Neeraj Shrivastava and Jyoti Bharti, "Multi-stage System for Preprocessing Mammograms", IEIE Transactions on Smart Processing and Computing, Vol. 9, Issue No. 2, April 2020, pp. 119-126

[25] R. Ramani, N. Suthanthira Vanitha, S. Valarmathy, "The Pre-Processing Techniques for Breast Cancer Detection in mammography Images", International Journal of Image, Graphics and Signal Processing, 2013 pp 47-54.

[26] Ashgan M. Omer, Mohammed Elfadil, "Preprocessing of Digital Mammogram Image Based on Otsu's Threshold", American Scientific Research Journal for Engineering Technology and Sciences (ASRJETS) (2017) Volume 37 No. 1, pp 220-229.

[27] J. Babu, S. Rangu, and P. Manogna, "A Survey on Different Feature Extraction and Classification Techniques Used in Image Steganalysis," Journal of Information Security, vol. 8, p. 186, 2017.
[28] J. Ma, C. Zou, and X. Jin, "An improved image enhancementalgorithm," Wuhan University Journal of Natural Sciences, vol. 22, pp. 85-92, 2017.

[29] S.-C. Pei, Y.-C. Zeng, and J.-J. Ding, "Color images enhancement using weighted histogram separation," in 2006 International Conference on Image Processing, 2006, pp. 2889-2892.

[30] Patil, P. P., \& Kotrappa, S. (2020). A Novel Approach to Detect Microcalcification for Accurate Detection for Diagnosis of Breast Cancer. In Internet of Things, Smart Computing and Technology: A Roadmap Ahead (pp. 81-94). Springer, Cham.

[31] Dey, N., Ashour, A. S., \& Bhattacharyya, S. (Eds.). (2020). Applied nature-inspired computing: algorithms and case studies. Springer Singapore.

[32] Bai, X., Zhou, F., \& Xue, B. (2012). Image enhancement using multi scale image features extracted by top-hat transform. Optics \& Laser Technology, 44(2), 328-336.

[33] Prathyusha Chalasani, S Rajesh. (2020). Lung CT Image Recognition using Deep Learning Techniques to Detect Lung Cancer. International Journal of Emerging Trends in Engineering Research. 3575-3579. 10.30534/ijeter/2020/113872020.

[34] B, Anilkumar. (2020). Tumor Classification using Block wise fine tuning and Transfer learning of Deep Neural Network and KNN classifier on MR Brain Images. International Journal of Emerging Trends in Engineering Research. $8 . \quad 574-583$. 10.30534/ijeter/2020/48822020.

[35] K. Deepak et.al. (2020). Cervical Cancer Classification. International Journal of Emerging Trends in Engineering Research. 8. 804-807. 10.30534/ijeter/2020/32832020.

[36] Amal Fouad et. al.(2019). MRI Brain Cancer Diagnosis Approach Using Gabor Filter and Support Vector Machine. International Journal of Emerging Trends in EngineeringResearch.907-914.10.30534/ijet er/2019/297122019. 